\title{
Propriedades psicométricas do Teste de Positividade de Fredrickson em um contexto militar
}

\author{
Livia Santos Ferreira ${ }^{1}$ \\ Nayara Baptista Silva ${ }^{1}$ \\ Rodolfo de Castro Ribas Junior ${ }^{1}$ \\ Marcos Aguiar de Souza ${ }^{1}$ \\ Thaynara Carvalho de Lima \\ ${ }^{1}$ Universidade Federal do Rio de Janeiro, RJ, Brasil \\ ${ }^{2}$ Universidade Federal Rural Rio de Janeiro, RJ, Brasil
}

\begin{abstract}
Resumo
A importância dos afetos para a adaptabilidade a circunstâncias adversas tem sido amplamente discutida na literatura, sendo enfatizado o papel central que exercem para o funcionamento psicológico. O presente estudo teve como objetivo validar, para o Brasil, o Teste de Positividade, desenvolvido por Fredrickson (2009). O instrumento possui 20 itens igualmente divididos em dois fatores: afetos positivos e afetos negativos. Participaram do estudo 1097 cadetes do curso de Oficiais do Exército. Foram realizadas duas coletas de dados, na primeira, foram reunidos 354 cadetes; na segunda, 743. Com os dados da primeira coleta realizou-se uma Análise Fatorial Exploratória, que confirmou a estrutura original do instrumento. A Análise Fatorial Confirmatória, realizada com os dados da segunda coleta, corroborou o modelo original do instrumento. Encontrou-se correlações positivas significativas entre esta medida e as de satisfação com a vida e com o trabalho. Os resultados demonstram a adequação do instrumento em contextos militares.
\end{abstract}

Palavras-chave: Afeto; Teste de Positividade; Militares.

\section{Psychometric properties of Fredrickson's Positivity Test in a military context}

\begin{abstract}
The role of affections for adaptability to adverse circumstances has been widely discussed in the literature, emphasizing the central role they play in psychological functioning. The present study aimed to validate, for Brazil, the Positivity Test, developed by Fredrickson (2009). The instrument has 20 items equally divided into two factors: positive affects and negative affects. This study included 1097 cadets of the course of army officers. There were two data collection; in the first one, 354 cadets participated, in the second, 743. With the first collection data held an Exploratory Factor Analysis which confirmed the original structure of the instrument. The Confirmatory Factor Analysis, performed with data from the second collection, corroborated the original model of the instrument. There were significant positive correlations between this measure and satisfaction with life and work. The results demonstrate the suitability of the instrument in military contexts.
\end{abstract}

Keywords: Affection; Test of Positivity; Military.

\section{Propiedades psicométricas de la Prueba de Positividad de Fredrickson en un contexto militar}

Resumen

El papel de los afectos para la adaptabilidad a las circunstancias adversas ha sido discutido en la literatura, siendo enfatizado el papel que ejercen para el funcionamiento psicológico. El presente estudio ha tenido como objetivo validar, para Brasil, el Test de Positividad, desarrollado por Fredrickson (2009). El instrumento tiene 20 ítems divididos en dos factores: afectos positivos y afectos negativos. Fueron entrevistados 1097 cadetes del curso del Ejército. En la primera recolección de datos se reunieron 354 cadetes; en la segunda, 743. Con los datos de la primera recolección se realizó un Análisis Factorial Exploratorio que confirmó la estructura original del instrumento. El Análisis Factorial Confirmatorio, realizado con los datos de la segunda recolección, corroboró el modelo original del instrumento. Se han encontrado correlaciones positivas significativas entre esta medida y las de satisfacción con la vida y con el trabajo. Los resultados demuestran la adecuación del instrumento en contextos militares.

Palabras clave: Afecto; Prueba de Positividad; Militares. 


\section{Introdução}

A importância dos afetos positivos e negativos para a adaptabilidade a circunstâncias adversas tem sido amplamente discutida na literatura, sendo enfatizado o papel central que exercem para o funcionamento psicológico (e.g., Coetzee \& Harry, 2014; Fredrickson, 1998; Seligman \& Csikszentmihalyi, 2000).

O principal objetivo deste estudo é avaliar a adequação do Teste de Positividade, desenvolvido por Fredrickson (2009), para o contexto de formação de oficiais do exército brasileiro. A relevância da investigação da vivência de emoções positivas e negativas em um contexto militar de formação é justificada uma vez que, de acordo com Cole (2014), o ambiente militar é marcado por fortes restrições, regras e hierarquias, sendo considerado por muitos como um ambiente naturalmente estressante. Assim, o estudo poderá contribuir para investigações futuras que permitam identificar o papel exercido por afetos positivos e negativos no contexto militar, principalmente no que se refere ao fator de proteção exercido por emoções positivas.

A palavra afeto tem sido normalmente utilizada para representar a variedade de estados de ânimo, sentimentos e emoções experimentados por uma pessoa, sendo eles agradáveis ou desagradáveis (Fredrickson \& Losada, 2005). Quando a sensação final é avaliada como agradável, chamamos afeto positivo entusiasmo, alegria e relaxamento. No caso contrário, quando a sensação final é avaliada como desagradável, chamamos afeto negativo - raiva, medo e tristeza (e.g., Zajonc, 1980; Watson, Clark, \& Tellegen, 1988; Pires, Filgueiras, Ribas, \& Santana, 2013). Os afetos podem ser entendidos como reações do organismo e cumprem importante papel na formação das emoções mais complexas (Pires et al., 2013).

Pesquisas apontam que a prevalência de afetos positivos traz benefícios para as relações sociais, para o ambiente de trabalho, para a saúde mental e física (Seligman \& Csikszentmihalyi, 2000; Wilson et. al, 2017; Elfenbein, 2014; Bartel \& Saavedra, 2000). De fato, a prevalência dos afetos positivos tem sido associado a uma melhor saúde física e mental (e.g., Salovey, Rothman, Detweiler, \& Steward, 2000) e também ao aumento da expectativa de vida (e.g., Danner, Snowdon, \& Friesen, 2001; Salovey, Rothman, Detweiler, \& Steward, 2000). Há indícios, inclusive, de que afetos positivos estariam associados ao controle do vírus HIV em mulheres (Wilson et. al, 2017). Cabe ressaltar que os afetos positivos estão também associados a maiores níveis de bem-estar subjetivo (Scheier \& Carver, 1993; Kuppens \& Realo, 2008).
Além disso, alguns autores apontam que o componente afetivo do bem-estar subjetivo depende principalmente da frequência e não da intensidade de experiências afetivas positivas e negativas (e.g., Kuppens \& Realo, 2008).

Evidências também indicam que a vivência de afetos positivos amplia o alcance de processos como atenção e cognição, promovendo importantes recursos físicos, intelectuais e sociais (e.g., Seligman \& Csikszentmihalyi, 2000; Wilson et al., 2017; Elfenbein, 2014; Bartel \& Saavedra, 2000). Além disso, tem sido apontado que os afetos positivos produzem benefícios não só no presente, funcionando também como um fator de proteção em situações futuras (Teixeira et al., 2014). Essas evidências permitem concluir que o estudo do afeto se torna relevante não só na vida em geral, mas também em contextos específicos, como na educação e no trabalho.

No âmbito do trabalho, evidências indicam que os afetos são usualmente compartilhados pelos membros de uma equipe, influenciando a qualidade do trabalho que realizam juntos (e.g., Elfenbein, 2014; Bartel \& Saavedra, 2000). Estudos indicam ainda que tanto a satisfação quanto o bem-estar no trabalho estão associados a estados afetivos (e.g., Martins \& Santos, 2006; Paschoal \& Tamayo, 2008).

A Positividade é um dos construtos estudados dentro da lógica da Psicologia Positiva, e diz respeito à disposição geral de um indivíduo para avaliar os diversos âmbitos da vida de forma positiva (Borsa, Damásio, \& Koller, 2016; Caprara et al., 2012). A Psicologia Positiva, por sua vez, é um ramo da psicologia que defende a concentração de esforços no estudo da saúde e dos elementos que a promovem em detrimento da doença, afirmando que esta mudança de foco pode gerar aumento da qualidade de vida, da longevidade, da redução dos custos de saúde e da melhoria na saúde mental (e.g., Seligman \& Csikszentmihalyi, 2000; Jayawickreme \& Pawelski, 2012).

Dentro deste contexto, o ambiente militar merece especial atenção, na medida em que é caracterizado por atividades que vêm sendo classificadas por diversos autores como naturalmente estressantes (e.g., Souza, 2015; Gaillard \& Soeters, 2006). A cultura militar é marcada por forte hierarquia, senso de regras e restrições (Cole, 2014). Tendo em vista que a prevalência de afetos positivos influencia as reações frente ao estresse (ver, e.g., Borsa, Damásio, \& Koller, 2016; Scheier \& Carver, 1993), é pertinente investigar o papel exercido pela positividade em um contexto de formação militar.

Outro ponto a ser destacado é que, considerando que o contexto militar se diferencia significativamente de outros contextos, principalmente daqueles em que 
muitos dos instrumentos têm sido desenvolvidos, estudos de validação de instrumentos se mostram particularmente importantes. Nos termos de Wilkinson et al. (1999) não se pode afirmar que "um teste não é confiável ou não confiável" (p. 596), uma vez que a confiabilidade diz respeito aos escores fornecidos por um teste (ou instrumento de medida) em uma dada amostra e não ao teste em si. Então, é crucial que um pesquisador investigue o instrumento que está fazendo uso, verificando suas qualidades psicométricas no contexto em que está sendo utilizado.

Para Jingkai (2015), sob influência da positividade, um líder militar poderá ter melhor desempenho, com maior receptividade à informação do ambiente e das pessoas, maior capacidade de integração essa informação e melhor uso da criatividade. Entretanto, destaca o papel da negatividade, de modo que é importante reduzi-la, mas não a eliminar, devido à sua importância para nossa sobrevivência. O medo, por exemplo, parece ser útil para nossa habilidade em detectar predadores e outras ameaças do ambiente.

A AMAN, em sua proposta pedagógica, visa, em quatro anos de formação, somados a um ano anterior na Escola Preparatória de Cadetes do Exército, preparar seus cadetes para o desempenho das funções do futuro oficial, capaz de adaptar suas ordens à realidade da situação mutável em que se encontra. Nesta formação, prevê ações voltadas ao desenvolvimento das áreas atitudinal, cognitiva e psicomotora. O currículo do Curso visa conciliar o ensino acadêmico (base científica e humanística) ao ensino militar (conhecimentos técnico-profissionais) com ênfase no desenvolvimento de conteúdos atitudinais, como liderança e chefia. O curso busca preparar o oficial da atualidade para que seja empregado em operações altamente descentralizadas, devendo decidir de forma acertada e rápida (Brasil, 2015).

Ao longo do curso o cadete participa de muitas atividades em campanha, nos diversos ambientes operacionais (selva, montanha, ambiente urbano, dentre outros) que o preparam para atuar em distintos contextos. Esta peculiar formação pode ser percebida, por alguns indivíduos, como exigente, desafiadora ou causadora de estresse e vulnerabilidade, fazendo com que os sujeitos tendam a fazer algo de modo a dominar a situação ou controlar as reações emocionais provocadas por um acontecimento (Patterson, 2016).

Um dos instrumentos utilizados para medir a positividade foi desenvolvido por Fredrickson (2009) e denominado Teste de Positividade. Este teste baseou-se em vários estudos sobre as relações entre o afeto positivo e o florescimento humano (e.g., Fredrickson \& Losada, 2013). Para os autores, flo- rescimento significa viver em um contexto ótimo de funcionamento humano, que engloba bondade, generatividade, crescimento e resiliência.

Fredrickson e Losada (2013) sugerem que a saúde mental está associada a uma proporção maior de afetos positivos em comparação com afetos negativos. A esta proporção, deu-se o nome de rácio de positividade. Trata-se de um questionário composto por 20 itens, dentre os quais 10 itens referem-se a afetos positivos e 10 a afetos negativos. Os participantes respondem a cada item utilizando uma escala de 0 a 4 , onde 0 equivale a "Nada" e 4 equivale a "Extremamente" e devem indicar a intensidade de emoção que experienciou nas últimas vinte e quatro horas. Para calcular o valor da rácio de positividade, deve-se somar os escores de afetos positivos, de afetos negativos, e dividir o valor total de afetos positivos pelo valor total de afetos negativos. Nessa publicação Fredrickson (2009) não apresenta propriedades psicométricas do Teste de Positividade.

Uma revisão de literatura revelou que o Teste de Positividade (Fredrickson, 2009) vem sendo utilizado em países como Portugal, embora apenas dissertações e teses tenham sido identificados (Becalli, 2014; Neves, 2016; Carvalho, 2014; Semedo, 2010).

Semedo (2014) realizou uma investigação acerca das experiências emocionais de 100 estudantes africanos no primeiro ano do Ensino Superior em Portugal e concluiu que o processo de integração social e acadêmica dos alunos está relacionado com uma maior rácio de positividade. Os resultados mostraram correlação positiva e significativa da rácio de positividade com a dimensão pessoal $(\mathrm{r}=0,32$; $p=0,00)$ e com a dimensão interpessoal/social $(\mathrm{r}=0,22 ; p=0,03)$ da integração social e acadêmica. Semedo (2014) reportou ainda os seguintes níveis de consistência interna (alfa de Cronbach) para dimensões positiva e negativa: 0,75 e 0,73 , respectivamente.

Um estudo exploratório realizado por Carvalho (2014) sobre bem-estar com 263 indivíduos (141 mulheres e 122 homens) mostrou correlação positiva e significativa entre os afetos positivos, satisfação com a vida, florescimento, coping pró-ativo e felicidade no último mês (Carvalho, 2014). Os resultados demonstraram que a satisfação com a vida $(r=0,59$; $p=0,001)$, o florescimento $(\mathrm{r}=0,42 ; p=0,001)$, os afetos positivos $(\mathrm{r}=0,49 ; p=0,001)$ e o coping próativo $(\mathrm{r}=0,35 ; p=0,001)$ se correlacionam de forma significativa e positiva com a felicidade no último mês. Em contrapartida, os afetos negativos ( $\mathrm{r}=-0,33$; $p=0,001)$ correlacionam-se de forma negativa e significativa com a felicidade no último mês, satisfação com a vida $(\mathrm{r}=-0,25 ; p=0,001)$, florescimento 
$(\mathrm{r}=-0,278 ; \mathrm{p}=0,001)$ e coping pró-ativo $(\mathrm{r}=-0,29$; $p=0,001)$.

Guzman e Gom (2013), conduziram um estudo psicométrico de uma versão do Teste de positividade de Fredrickson (2009). Os autores aplicaram uma versão em espanhol em 383 estudantes universitários (84\% mulheres, $16 \%$ homens) com idades entre 17 e 43 anos $(M=20,69)$. Os autores conduziram uma análise fatorial exploratória (Kaiser-Meyer-Olkin $=85$; Teste de Esfericidade de Bartlett $=2799,705, p=0,001)$ e identificaram 3 fatores (vivência de afeto positivo, vivência de afeto negativo, e percepção de afeto negativo. Os alfas reportados para os 3 fatores foram, respectivamente, 0,$90 ; 0,78 ; 0,74$.

Foi conduzida uma busca bibliográfica de estudos sobre positividade no contexto militar brasileiro. A plataforma utilizada para esta busca foi o Scielo Brasil. As palavras-chave utilizadas foram: positividade, teste de positividade, militar. Não foi encontrado nenhum artigo sobre essa escala em contextos brasileiros, inclusive o contexto militar. Sendo assim, este artigo visa validar o Teste de Positividade para o Brasil, especificamente, para o contexto militar. E, posteriormente, estudar o papel da positividade na qualidade de vida de militares em formação.

O presente trabalho foi conduzido com o objetivo de investigar a consistência interna e confiabilidade do Teste de Positividade, e sua validade e adequação para um contexto brasileiro. Especificamente, buscou-se avaliar a adequação da medida para o contexto de formação do oficial do exército brasileiro. Adicionalmente, buscou-se avaliar associações entre Positividade, Satisfação com a Vida e Satisfação com o Trabalho. Estas variáveis foram escolhidas pelo fato de serem indicadoras de qualidade de vida, sendo de vital importância em um contexto de formação militar. Assim, espera-se encontrar correlação positiva entre afetos positivos, satisfação com a vida e satisfação com o trabalho.

\section{Método}

\section{Participantes}

Participaram do estudo um total de 1097 cadetes dos quatro anos de formação do curso de Oficiais do Exército, na Academia Militar das Agulhas Negras, todos do sexo masculino, com idade variando de 17 a 23 anos (média de 19,32 anos; $d p=1,23$ anos) que preencheram o instrumento de pesquisa disponibilizado de forma digital na Intranet da instituição. Todos os participantes assinaram um Termo de Consentimento Livre e Esclarecido redigido de acordo com os critérios do Comitê de Ética em Pesquisa da Universidade
Federal Rural do Rio de Janeiro, que aprovou este projeto (no 557/2015).

\section{Instrumentos}

Teste de Positividade: foi utilizada a versão em português do Teste de Positividade (Positivity Self Test, PST; Fredrickson, 2009). Esta escala foi idealizada para avaliar a vivência de afetos positivos e negativos e a razão ou rácio de positividade. A seguinte instrução é dada aos participantes: "Com este questionário pretendemos saber como se sentiu no último mês. Usando a escala abaixo, indique quanto experienciou os seguintes sentimentos, colocando um número a seguir a cada item: O teste é composto por 10 itens sobre afetos positivos (e.g., "O quanto divertido, cômico ou engraçado se sentiu?") e por 10 itens sobre afetos negativos ("O quanto triste, desanimado ou infeliz se sentiu?". Cada item foi avaliado em uma escala de 4 pontos ( 0 - nada; 1 - um pouco; 2 - moderadamente; 3 - bastante; 4 - extremamente). A autora não reportou propriedades psicométricas no estudo original.

Escala de Satisfação com a Vida (SWLS; Diener, Emmons, Larsen, \& Griffin, 1985; versão brasileira adaptada por Gouveia, Milfont, Fonseca, \& Coelho, 2009). A versão brasileira, conforme a escala original, conta com cinco afirmativas, cujas respostas variam em uma escala Likert de 1 (discordo totalmente) a 7 (concordo totalmente). No estudo de Gouveia et al. (2009), com uma amostra de 2180 participantes encontrou-se resultados que confirmaram a estrutura unifatorial e confiabilidade desta versão da escala para uso no Brasil ( $\chi^{2} / \mathrm{df:} 9.00$, GFI: 0.99, RMSEA: 0.060, Coeficiente de Alfa de Cronbach: 0.81 na amostra geral). No presente estudo o Alfa de Cronbach observado foi de 0,79 .

Para mensuração da satisfação com o trabalho, foi utilizada uma versão em português da Job Satisfaction Scale desenvolvida por Judge e Klinger (2000). A escala é composta de cinco itens sendo que cada item foi avaliado em uma escala de cinco níveis ( 1 = Nunca; 2=Algumas Vezes; 3=Regularmente; 4=Bastantes Vezes; 5= Sempre). Judge e Klinger reportaram um Coeficiente de Alfa de Cronbach de 0,80 no estudo original. No presente estudo o Alfa de Cronbach observado foi de 0,74 .

Questionário de dados sociodemográficos. Um questionário estruturado contendo informações sobre idade, sexo, escolaridade, emprego e religião foi utilizado.

\section{Procedimentos}

Os dados foram coletados em dois momentos, com intervalo de 2 semanas. Na primeira coleta, foram 
reunidos 354 cadetes e na segunda, 743 cadetes. A participação foi voluntária e todos os preceitos éticos foram seguidos.

\section{Resultados}

Os dados obtidos na primeira coleta (354 cadetes) foram utilizados para a Análise Fatorial Exploratória dos principais eixos com rotação oblíqua, que confirmou a estrutura original do instrumento, com dois fatores. Foram obtidos indicativos satisfatórios para a análise $(\mathrm{KMO}=0,92$; teste de esfericidade de Bartlett significativo ao nível de 0,01 ; variância média explicada de $48,10 \%$, e consistência interna (Alfa de Cronbach) dos fatores de 0,90 para emoções positivas e 0,90 para emoções negativas).

Uma análise fatorial confirmatória (Figura 1) foi realizada com os 743 cadetes que participaram da segunda coleta de dados. Os resultados corroboram o modelo original do instrumento, sendo obtidos os índices recomendados na literatura (Tabela 1) (Hair et al., 2005; Marôco, 2010).
Apesar dos valores apresentados tais resultados indicam a adequação da Escala de positividade para mensuração a vivência de afetos positivos e negativos no contexto de formação do Oficial do Exército Brasileiro. O uso do instrumento permitirá o desenvolvimento de estudos que permitam melhor compreender o papel da vivência de afetos positivos e negativos para o desenvolvimento de recursos que permitam melhor lidar com os desafios da vida militar, especialmente durante o processo de formação.

Os benefícios do predomínio dos afetos positivos e da existência de afetos negativos (em menor grau) têm sido amplamente narrados em contextos militares (Jingkai, 2015; Faulk et al., 2012; Fredrickson et al, 2000). Entretanto, em contextos militares brasileiros são escassos os estudos sobre o tema, situação que poderá se modificar com a existência de um instrumento validado para uso em organizações militares. Realizou-se análises descritivas para observar os escores dos participantes nas medidas de Afetos Positivos, Afetos Negativos, Satisfação com o Trabalho e Satisfação com a vida, os resultados podem ser observados na Tabela 2 .

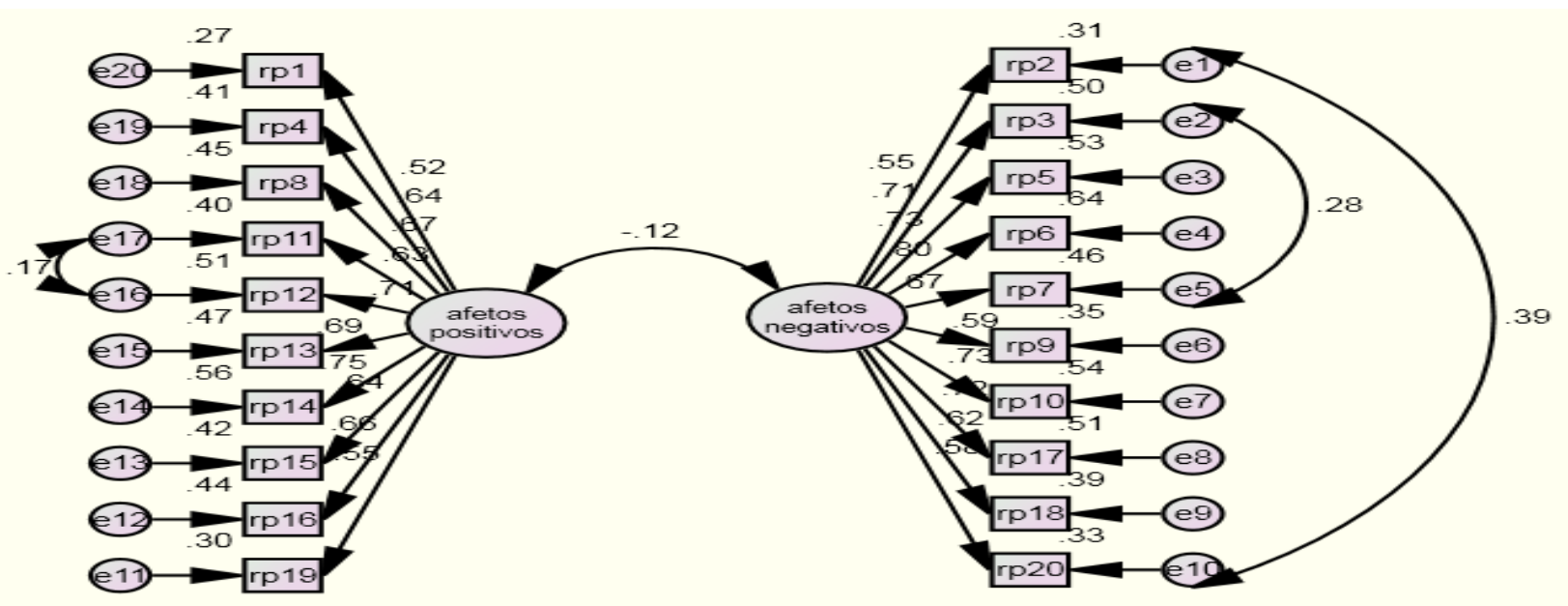

Figura 1. Análise Fatorial Confirmatória da Escala de Positividade

TABELA 1

Parâmetros da análise fatorial confirmatória da Escala de Positividade

\begin{tabular}{lcc}
\hline Indicadores & Valor obtido & Valor ideal \\
$\chi^{2}$ & 418,81 & - \\
$\chi^{2} / \mathrm{gl}$ & 2,64 & $<5$ \\
$\mathrm{RMR}$ & 0,45 & $<0,05$ \\
GFI & 0,95 & $\geq 0,90$ \\
AGFI & 0,92 & $\geq 0,90$ \\
CFI & 0,95 & Próximo a 1 \\
RMSEA & $0,05(0,04-0,07)$ & $<0,10$ \\
\hline
\end{tabular}

TABELA 2

Média, mediana e desvio padrão dos escores obtidos pelos participantes nas medidas de Afetos Positivos, Afetos Negativos, Satisfação com o Trabalho e Satisfação com a vida

\begin{tabular}{lccc}
\hline & Média & Mediana & $\begin{array}{c}\text { Desvio } \\
\text { Padrão }\end{array}$ \\
Afetos Negativos & 2,35 & 2,20 & 0,77 \\
Afetos Positivos & 3,35 & 3,40 & 0,69 \\
Satisfação com o trabalho & 3,62 & 3,67 & 0,77 \\
Satisfação com a vida & 3,54 & 3,60 & 0,68 \\
\hline
\end{tabular}


Visando obter indicativos adicionais de validade da Escala de Positividade em contexto militar, foi realizado um estudo correlacional, sendo consideradas a satisfação com o trabalho e a satisfação com a vida. Os resultados obtidos na correlação entre afetos positivos e afetos negativos e satisfação com o trabalho e com a vida, conforme pode ser observado na Tabela 3, estão de acordo com o previsto na literatura e de maneira geral confirma o resultado de diversos estudos na área.

\section{TABELA 3}

Análise correlacional de afetos positivos, afetos negativos, satisfação com o trabalho e satisfação com a vida

\begin{tabular}{lcccc}
\hline & 1 & 2 & 3 & 4 \\
1. Afetos Positivos & - & & & \\
2. Afetos Negativos & $-0,19^{* *}$ & - & & \\
3. Satisfação com o trabalho & $0,52^{* *}$ & $-0,23^{* *}$ & - & \\
4. Satisfação com a vida & $0,45^{* *}$ & $-0,40^{* *}$ & $-0,53^{* *}$ & - \\
\hline
\end{tabular}

** Significativo ao nível de 0,01 .

\section{Considerações finais}

O principal objetivo do presente estudo foi a validação da escala de positividade para o contexto de formação do oficial do exército brasileiro. Apesar desse objetivo, é importante considerar que o uso da escala em outros contextos militares brasileiros irá requerer o mesmo esforço, de modo a verificar a adequação do instrumento para estudos seja com militares em contexto de atuação profissional, seja com mili- tares de outras patentes ou mesmo militares de outras Forças.

A vivência de afetos positivos e negativos, bem como a consideração da psicologia positiva em contextos militares, tem permitido importantes progressos no sentido de promoção de saúde e qualidade de vida de militares, sobretudo em contextos de formação. E considerando que tais militares serão os futuros líderes, os benefícios dessa linha de investigação podem ser potencializados.

De maneira geral é possível afirmar que os objetivos do estudo foram atingidos. Assim, a validação da escala de racio de positividade de Fredrickson para amostras militares no contexto brasileiro contribui para o desenvolvimento de estudos na área e da melhor compreensão do papel de vivência de afetos positivos e negativos para a saúde e qualidade de vida no contexto militar.

Além da validade da escala como atestada no presente estudo, a correlação com a satisfação com o trabalho e a satisfação com a vida fornecem bons indicativos de validade externa do instrumento. Em termos de aplicabilidade dos resultados para o contexto investigado, é possível dizer que a vivência de afetos positivos traz importantes contribuições para a formação do oficial do Exército Brasileiro.

Investigações futuras podem proporcionar melhor compreensão dos afetos positivos, dos afetos negativos e do balanço entre ambos para a saúde, qualidade de vida e desempenho de militares brasileiros tanto em contextos de formação como em contextos de atuação profissional.

\section{Referências}

Bartel, C. A. \& Saavedra, R. (2000). The collective construction of work group moods. Administrative Science Quarterly, 45(2), 197-231. https://doi.org/10.2307/2667070

Becalli, M. S. F. S. (2012). Caminho para o Florescimento: satisfação com a vida, emoções positivas e otimismo em adultos. Dissertação [Mestrado], Universidade Lusófona de Humanidades e Tecnologias, Lisboa.

Brasil (2015). Diretoria de Educação Superior Militar (DESMil), Boletim Interno no 18, de 12 de março de 2015. Aprova o Regimento Interno da Academia Militar das Agulhas Negras. Regimento Interno da Academia Militar das Agulhas Negras, Rio de Janeiro, RJ, 12 mar. 2015.

Carvalho, V. S. T. (2014). A Felicidade e o Coping Pró-Ativo: Estudo Exploratório em Homens e Mulheres. Dissertação [Mestrado], Universidade Lusófona de Humanidades e Tecnologias, Lisboa.

Coetzee, M. \& Harry, N. (2014). Emotional intelligence as a predictor of employees' career adaptability. Journal of Vocational Behavior, 84(1), 90-97. https://doi.org/10.1016/j.jvb.2013.09.001

Cole, R. F. (2014). Understanding Military Culture: A Guide for Professional School Counselors. The Professional Counselor, 4(5), 497-504. https://doi.org/10.15241/tpc.4.5.413

Danner, D. D., Snowdon, D. A., \& Friesen, W. V. (2001). Positive emotions in early life and longevity: Findings from the nun study. Journal of Personality and Social Psychology, 80(5), 804-813. https://doi.org/10.1037//0022-3514.80.5.804

Delahaij, R., Gaillard, A. W. K., \& Soeters, J. M. L. M. (2006). Stress Training and the New Military Environment. RTO.

Diener, E, Emmons, R. A., Larsen, R. J., \& Griffin, S. (1985). The satisfaction with life scale. Journal of Personality, 49(1), 71-75. https://doi.org/10.1207/s15327752jpa4901_13 
Diener, E. \& Emmons, R. A. (1985). The Independence of Positive and Negative Affect. Journal of Personality and Social Psychology, 47(5), 1105-1117. https://doi.org/10.1037/0022-3514.47.5.1105

Diener, E. (2012). New Finding sand Future Directions for Subjective Well-Being Research. American Psychologist, 67(8), 590-597. https://doi.org/10.1037/a0029541

Diener, E., Inglehart, R., \& Tay, L. (2012). Theory and Validity of Life Satisfaction Scales. Social Indicators Research, $112(3), 497-527$. https://doi.org/10.1007/s11205-012-0076-y

Elfenbein, H. A. (2014). The many faces of emotional contagion: An affective process theory of affective linkage. Organizational Psychology Review, 4(4), 326-362. https://doi.org/10.1177/2041386614542889

Faulk, K.E.; Gloria, C.T.; Cance, J. D., \& Steinhardt, M. A. (2012). Depressive Symptoms among US Military Spouses during Deployment: The Protective Effect of Positive Emotions. Armed Forces \& Society, 38(3) 373-390. https://doi. org/10.1177/0095327X11428785

Fredrickson, B. L. (1998). What good are positive emotions? Review of General Psychology, 2, 300-319. https://doi. org/10.1037/1089-2680.2.3.300

Fredrickson, B. L. (2009). Positivity: Ground breaking research reveal show to embrace the hidden strength of positive emotions, overcome negativity, and thrive. New York: Crown.

Fredrickson, B. L. \& Losada, M. F. (2013). Positive Affect and the Complex Dynamics of Human Flourishing. American Psychologist, 68(9), 822-822. https://doi.org/10.1037/a0034435

Fredrickson, B. L., Mancuso, R. A., Branigan, C., \& Tugade, M. M. (2000). The Undoing Effect of Positive Emotions. Motivation and Emotions, 24(4), 237-258. https://oi.org/10.1023/A:1010796329158

Gouveia, V. V., Milfont, T., Fonseca, P. N., \& Coelho, J. A., P. M. (2009). Life satisfaction in Brazil: Testing the psychometric properties of the Satisfaction With Life Scale (SWLS) in five Brazilian samples. Social Indicators Research, 90(2), 267-277. https://doi.org/10.1007/s11205-008-9257-0

Guzman, L. R. C., Gom, S. B. (2013). Traducción al español y propiedades psicométricas del instrumento Positivity Self Test. Psicología Iberoamericana, 21(1), 53-64. (DOI inexistente)

Hair Jr., Joseph F; Anderson, R. E.; Tatham, R. L., \& Black, W. C. (2005) . Análise multivariada de dados. Porto Alegre: Artmed.

Jayawickreme, E., \& Pawelski, J. O. (2012). Positivity and the capabilities approach. Philosophical Psychology, 26(3), 383-400. https://doi.org/10.1080/09515089.2012.66068

Jingkai, C. (2015). The Emotional Fitness of a Soldier - Balancing Positivity and Negativity in the Military. POINTER Journal of the Singapore Armed Forces, 41(3), 64-77.

Judge, T. A.; Klinger, R. (2008). Job satisfaction: Subjective well-being at work. In Michael Eid \& Randy J. Larsen (Ed.). The science of subjective well-being (pp. 393-413). New York: Guilford Press.

Judge, T. A. \& Kammeyer-Mueller, J.D. (2012). Job Attitudes. Annual Review of Psychology, 63, 341-367. https://doi. org/10.1146/annurev-psych-120710-100511

Kuppens, P., Realo, A., \& Diener, E. (2008). The role of positive and negative emotions in life satisfaction judgment across nations. Journal of Personality and Social Psychology, 95(1), 66-75. https://doi.org/10.1037/0022-3514.95.1.66

Marôco, J. (2010). Análise de equações estruturais. Lisboa: Report Number.

Martins, M., Santos, G. (2006). Adaptação e Validação de Construto da Escala de Satisfação no Trabalho. Psico-USF, 11(2), 195-205. https://doi.org/10.1590/S1413-82712006000200008

Neves, S. M. S. M. (2016). As Emoções ao Longo do Ciclo de Vida. Tese [Doutorado], Universidade Lusófona de Humanidades e Tecnologias, Lisboa.

Paschoal, T., Tamayo, A. (2008). Construção e Validação da Escala de Bem-Estar no trabalho. Avaliação Psicológica, 7(1), 11-22.

Patterson, G. T. (2016). A Brief Exploratory Report of Coping Strategies Among Police Recruits During Academy Training. Psychological Reports, 119(2), 557-567. https://doi.org/10.1177/0033294116662685

Pires, P., Filgueiras, A., Ribas, R. \& Santana, C. (2013). Funcionamento Diferencial de Itens (DIF) e experiência de afeto: questões de gênero. Gerais: Revista Interinstitucional de Psicologia, 6(1),114-126.

Salovey, P., Rothman, A. J., Detweiler, J. B., \& Steward, W. T. (2000). Emotional states and physical health. American Psychologist, 55(1), 110-121. https://doi.org/10.1037/0003-066X.55.1.110

Scheier, M. F. \& Carver, C. S. (1993). On the power of positive thinking: The benefits of being optimistic. Current Directions in Psychological Science, 2, 26-30. https://doi.org/10.1111/1467-8721.ep10770572

Seligman, M. E. P. \& Csikszentmihalyi, M. (2000). Positive psychology: An introduction. American Psychologist, 55 , 05-14. https://doi.org/10.1037/0003-066X.55.1.5

Semedo, M. A. L. (2010). Emoções Mistas: Integração Social e Académica dos Alunos Provenientes dos PALOP. Dissertação [Mestrado], Universidade Lusófona de Humanidades e Tecnologias, Lisboa.

Souza, Y. R. (2015). Estresse e estratégias de “coping” em treinamentos militares extenuantes na selva amazônica. Dissertação [Mestrado], Programa de Mestrado em Psicologia, Universidade Federal de Rondônia, Porto Velho, RO.

Spector, P. (2012). Psicologia nas Organizações (4⿳亠丷⿵冂丶 ed.). São Paulo: Saraiva. 
Teixeira, M. T. V., Seraceni, M. F., Suriano R., Santana, N. Z., Carreiro, L. R., \& Paula, C. S. (2014). Fatores de proteção associados a problemas emocionais e comportamentais em escolares. Estudos de Psicologia, 31(4), 539-548. https:// doi.org/10.1590/0103-166X2014000400008

Watson, D. \& Clark, L. A. (1988). Development and validation of brief measures of positive and negative affect: The PANAS scales. Journal of Personality and Social Psychology, 54(6), 1.063-1.070. https://doi.org/10.1037//00223514.54.6.1063

Wilkinson, L. \& Task Force on Statistical Inference (1999). Statistical methods in psychology journals. American Psychologist, 54, 594-604. https://doi.org/10.1037/0003-066X.54.8.594

Wilson, T. E., Weedon, J., Cohen, M. H., Golub, E. T., Milam, J., Young, M. A., \& Fredrickson, B. L. (2017). Positive Affect and Its Association With Viral Control Among Women With HIV Infection. Health Psychology, 36(1), 91-100. https://doi.org/10.1037/hea0000382

Zajonc, R. B. (1980). Feelings and thinking: preferences need no inferences. American Psychologist, 35(2), 151-175. https://doi.org/10.1037/0003-066X.35.2.151

Declaração de Contribuição:

Os autores Livia Santos Ferreira, Nayara Baptista Silva, Rodolfo de Castro Ribas Junior, Marcos Aguiar de Souza,

Thaynara Carvalho de Lima, declaram ser responsáveis pela elaboração do manuscrito intitulado "Propriedades psicométricas do Teste de Positividade de Fredrickson em um contexto militar", sendo que as duas primeiras autoras participaram da análise de dados, revisão de literatura, redação do artigo e preparação final do manuscrito para publicação e os três últimos autores participaram da concepção da pesquisa, coleta de dados, análise de dados, revisão de literatura e redação do artigo.

Declaramos, ainda, não haver conflitos de interesse.

Dados dos autores:

Livia Santos Ferreira - Doutoranda, Universidade Federal do Rio de Janeiro.

Nayara Baptista Silva - Mestranda, Universidade Federal do Rio de Janeiro.

Rodolfo de Castro Ribas Junior - Doutor, Universidade Federal do Rio de Janeiro.

Marcos Aguiar de Souza - Doutor, Universidade Federal do Rio de Janeiro.

Thaynara Carvalho de Lima - Mestranda, Universidade Federal Rural Rio de Janeiro.

\section{Endereço para correspondência:}

Livia Santos Ferreira

Rua Agostinho dos Santos, 202

21941-260 Rio de Janeiro, RJ, Brasil

$<$ liviasantosfer@gmail.com>

Recebido em: 04.10.2017

Aceito em: 16.10 .2018 